\title{
Effectiveness and safety of apixaban versus warfarin in non-valvular atrial fibrillation patients in "real-world" clinical practice
}

\section{A propensity-matched analysis of 76,940 patients}

\author{
Xiaoyan Li'; Steve Deitelzweig²; Allison Keshishian ${ }^{3}$; Melissa Hamilton'; Ruslan Horblyuk'; Kiran Gupta'; Xuemei Luo5; \\ Jack Mardekian4; Keith Friend ${ }^{1}$; Anagha Nadkarni'; Xianying Pan'; Gregory Y. H. Lip ${ }^{7,8}$ \\ ${ }^{1}$ Bristol-Myers Squibb Company, Lawrenceville, New Jersey, USA; ${ }^{2}$ Ochsner Clinic Foundation, Department of Hospital Medicine, New Orleans, Louisianna, USA and The University \\ of Queensland School of Medicine, Ochsner Clinical School, New Orleans, Louisianna, USA; ${ }^{3}$ STATinMED Research, Ann Arbor, Michigan, USA; ${ }^{4}$ Pfizer, Inc., New York, New York, \\ USA; ${ }^{5}$ Pfizer, Inc., Groton, Conneticut, USA; ${ }^{6}$ Bristol-Myers Squibb Company, Wallingford, Conneticut, USA; ${ }^{7}$ Institute of Cardiovascular Sciences, University of Birmingham, \\ Birmingham, UK; ${ }^{8}$ Aalborg Thrombosis Research Unit, Department of Clinical Medicine, Aalborg University, Aalborg, Denmark
}

\begin{abstract}
Summary
The ARISTOTLE trial showed a risk reduction of stroke/systemic embolism (SE) and major bleeding in non-valvular atrial fibrillation (NVAF) patients treated with apixaban compared to warfarin. This retrospective study used four large US claims databases (MarketScan, PharMetrics, Optum, and Humana) of NVAF patients newly initiating apixaban or warfarin from January 1, 2013 to September 30, 2015. After 1:1 warfarin-apixaban propensity score matching (PSM) within each database, the resulting patient records were pooled. Kaplan-Meier curves and Cox proportional hazards models were used to estimate the cumulative incidence and hazard ratios (HRs) of stroke/SE and major bleeding (identified using the first listed diagnosis of inpatient claims) within one year of therapy initiation. The study included a total of 76,940 (38,470 warfarin and 38,470 apixaban) patients. Among the 38,470 matched pairs, 14,563 were from MarketScan, 7,683 were from PharMetrics, 7,894 were from Optum, and 8,330 were from Humana. Baseline characteristics were balanced between the two cohorts with a mean (standard deviation [SD]) age of 71 (12) years and a mean (SD) $\mathrm{CHA}_{2} \mathrm{DS}_{2}$-VASc score of 3.2 (1.7). Apixaban initiators had a
\end{abstract}

Correspondence to:

Xiaoyan Li

Bristol-Myers Squibb

3401 Princeton Pike

Lawrenceville, NJ 08648, USA

Tel.: +1 6093024478

Email: shawn.li@bms.com

Note: The review process for this manuscript was fully handled by Christian

Weber, Editor in Chief.

Supplementary Material to this article is available online at

www.thrombosis-online.com. significantly lower risk of stroke/SE (HR: $0.67,95 \% \mathrm{Cl}: 0.59-0.76)$ and major bleeding (HR: $0.60,95 \% \mathrm{Cl}: 0.54-0.65$ ) than warfarin initiators. Different types of stroke/SE and major bleeding - including ischaemic stroke, haemorrhagic stroke, SE, intracranial haemorrhage, gastrointestinal bleeding, and other major bleeding - were all significantly lower for apixaban compared to warfarin treatment. Subgroup analyses (apixaban dosage, age strata, $\mathrm{CHA}_{2} \mathrm{DS}_{2}$-VASc or HAS-BLED score strata, or dataset source) all show consistently lower risks of stroke/SE and major bleeding associated with apixaban as compared to warfarin treatment. This is the largest "real-world" study on apixaban effectiveness and safety to date, showing that apixaban initiation was associated with significant risk reductions in stroke/SE and major bleeding compared to warfarin initiation after PSM. These benefits were consistent across various high-risk subgroups and both the standardand low-dose apixaban dose regimens.

\section{Keywords}

Apixaban, warfarin, non-valvular atrial fibrillation, stroke, major bleeding

\section{Financial support:}

This study was funded by Bristol-Myers Squibb Company and Pfizer, Inc.

Received: January 31, 2017

Accepted after minor revision: March 9, 2017

Epub ahead of print: March 16, 2017

https://doi.org/10.1160/TH17-01-0068

Thromb Haemost 2017; 117: 1072-1082

\section{Introduction}

Atrial fibrillation (AF) is recognised as the most common sustained cardiac arrhythmia in the United States and is associated with a nearly five-fold excess of stroke (1). AF prevalence in the United States was estimated at 5.2 million in 2010 and is projected to become 12.1 million in 2030, with an annual increase of $4.3 \%$ (2).
Vitamin K antagonists (VKAs; e.g. warfarin) have been the mainstay treatment in stroke prevention for AF patients for several decades, reducing stroke among AF patients by $64 \%$ compared to a control or placebo; however, an increased risk of major bleeding was observed when compared with no anticoagulant treatment or placebo $(3,4)$. In addition to a higher risk of major bleeding, warfarin treatment is hindered by its narrow therapeutic range, drug 
and food interactions, the requirement of regular blood test monitoring of the international normalised ratio (INR), and frequent need of dose adjustment (5).

The non-VKA oral anticoagulants (NOACs) - including dabigatran, rivaroxaban, apixaban, and edoxaban - were approved for stroke prevention in non-valvular atrial fibrillation (NVAF) patients by the US Food and Drug Administration in October 2010, November 2011, December 2012, and January 2015, respectively. Compared to warfarin, no anticoagulation monitoring is required and fewer drug and food interactions are evident (6). In Phase 3 clinical trials, all four NOACs are at least as efficacious and safe as warfarin (7-10). Apixaban is the only NOAC to show risk reduction in both stroke/systemic embolism (SE) and major bleeding compared to warfarin in its Phase 3 trial (ARISTOTLE) (9). Apixaban is used to treat NVAF in two dosing forms: $5 \mathrm{mg}$ and $2.5 \mathrm{mg}$. Apixaban $5 \mathrm{mg}$ is recommended to most patients, and the reduced apixaban $2.5 \mathrm{mg}$ is only recommended to patients who meet at least two of three criteria: age $\geq 80$ years, body weight $\leq 60 \mathrm{~kg}$, and serum creatinine $\geq 1.5 \mathrm{mg} / \mathrm{dl}$ (11).

"Real-world" observational studies have been conducted to estimate the effectiveness and safety of oral anticoagulants (OACs; including VKAs and NOACs) outside clinical trial settings. Since apixaban was approved only in December 2012, few studies have evaluated apixaban; prior "real-world" studies were limited by sample size and follow-up duration, particularly for effectiveness endpoints.

An a priori power calculation was completed based on the event rates observed in the ARISTOTLE trial and an estimated duration of follow-up in US claims datasets: an estimated 25,000 patients are needed to adequately examine the effectiveness of apixaban compared to warfarin (with an alpha of 0.05 and power of $80 \%$ ). Thus, to achieve the required sample size, this retrospective study was conducted using pooled data from four US claims datasets. The risk of primary endpoints in the ARISTOTLE trial (stroke/SE and major bleeding) was compared among treatmentnaïve NVAF patients who were prescribed warfarin or apixaban.

\section{Materials and methods}

\section{Data sources}

Data in this study were pooled from four large, nationally-representative claims databases in the US - Truven MarketScan ${ }^{\circledR}$ Commercial Claims and Encounter and Medicare Supplemental and Coordination of Benefits Database ("MarketScan") (12), IMS PharMetrics Plus ${ }^{\mathrm{Ta}}$ Database ("PharMetrics") (13), Optum Clinformatics " Data Mart (“Optum") (14), and Humana Research Database ("Humana") (15) - from January 1, 2012 to September 30, 2015. The four datasets include claims from over 163 million members of commercial and Medicare Advantage/supplemental plans. The datasets contain information on patient demographics and enrolment history as well as medical claims from inpatient hospital, outpatient hospital, emergency room, physician's office, and surgery centres. The medical claims are coded using International Classification of Disease, 9th Revision, Clinical Modifica- tion (ICD-9-CM), Current Procedural Terminology, or Healthcare Common Procedure Coding System codes. Pharmacy claims include the drug dispensed using the National Drug Code coding system. Although lacking clinical richness, these claims datasets provide access to the healthcare experience of millions of patients across continuum of care setting over a multiyear period of time, and have often been used in pharmacoepidemiology and pharmacovigilance studies, comparative effectiveness research, and health care economic analyses. These datasets have also been used in previous pooled analyses of various therapeutic areas (16-19), including one analysis using the MarketScan and Optum datasets on the comparative effectiveness and safety of dabigatran versus warfarin in NVAF patients (20). The Optum and Humana databases contain information of beneficiaries from unique insurance plans, which guarantees no duplicates on the health-plan level when pooled with other datasets. The other two datasets contain information from employer-provided health plans, with reported potential duplicates of only $0.5 \%$ in a study using both datasets (21).

\section{Patient selection}

NVAF patients who were aged $\geq 18$ years and had $\geq 1$ pharmacy claim for apixaban or warfarin during the identification period (January 1, 2013 to September 30, 2015) were included in the study. AF patients were identified using ICD-9-CM code 427.31, a validated code used to identify $\mathrm{AF}$ patients with a median positive predictive value of $89 \%$ (22). The date of the first apixaban or warfarin pharmacy claim during the identification period was designated as the index date. Patients were required to have the AF diagnosis before or on the index date and have continuous medical and pharmacy health plan enrolment for $\geq 12$ months prior to the index date.

Patients with evidence of valvular heart disease, venous thromboembolism, transient AF (pericarditis, hyperthyroidism, thyrotoxicity), or heart valve replacement/transplant during the 12 months prior to or on the index date, or with pregnancy during the study period were excluded. Patients treated with any OACs within 12 months before the index date or with $>1$ OAC on the index date were also excluded.

\section{Outcome measures}

The outcome measures were stroke/SE and major bleeding events identified using the first listed ICD-9-CM diagnosis of inpatient claims. The diagnosis codes used for stroke/SE and major bleeding were based on a validated administrative claim-based algorithm as well as the International Society on Thrombosis and Haemostasis' definition of major bleeding as used in the ARISTOTLE trial (Suppl. Table 1, available online at www.thrombosis-online.com) $(9,23,24)$. Stroke/SE was further stratified by ischaemic stroke, haemorrhagic stroke, and SE; major bleeding was further stratified by gastrointestinal (GI) bleeding, intracranial haemorrhage (ICH), and other major bleeding. To assess the outcomes, patients were followed from the day after the index date and were censored at the first outcome event, 30 days after the discontinuation date, the 
Table 1: Baseline characteristics for propensity score matched apixaban and warfarin patients.

\begin{tabular}{|c|c|c|c|c|}
\hline & \multicolumn{2}{|c|}{$\begin{array}{l}\text { Warfarin Cohort } \\
(\mathrm{N}=38,470)\end{array}$} & \multicolumn{2}{|c|}{$\begin{array}{l}\text { Apixaban Cohort } \\
(\mathrm{N}=38,470)\end{array}$} \\
\hline & N/Mean & $\% / S D$ & N/Mean & $\% / S D$ \\
\hline Age (years) & 70.9 & 11.9 & 70.9 & 12.0 \\
\hline $18-54$ & 3,304 & $8.6 \%$ & 3,203 & $8.3 \%$ \\
\hline $55-64$ & 8,942 & $23.2 \%$ & 8,962 & $23.3 \%$ \\
\hline $65-74$ & 10,660 & $27.7 \%$ & 10,665 & $27.7 \%$ \\
\hline$\geq 75$ & 15,564 & $40.5 \%$ & 15,640 & $40.7 \%$ \\
\hline \multicolumn{5}{|l|}{ Gender } \\
\hline Male & 23,015 & $59.8 \%$ & 22,946 & $59.7 \%$ \\
\hline Female & 15,455 & $40.2 \%$ & 15,524 & $40.4 \%$ \\
\hline \multicolumn{5}{|l|}{ US Geographic Region } \\
\hline Northeast & 5,911 & $15.4 \%$ & 5,949 & $15.5 \%$ \\
\hline Midwest & 10,264 & $26.7 \%$ & 10,337 & $26.9 \%$ \\
\hline South & 16,186 & $42.1 \%$ & 16,146 & $42.0 \%$ \\
\hline West & 5,817 & $15.1 \%$ & 5,739 & $14.9 \%$ \\
\hline Other & 292 & $0.8 \%$ & 299 & $0.8 \%$ \\
\hline \multicolumn{5}{|l|}{ Baseline Comorbidity } \\
\hline $\begin{array}{l}\text { Deyo-Charlson Comorbidity } \\
\text { Index Score }\end{array}$ & 2.5 & 2.5 & 2.5 & 2.4 \\
\hline $\mathrm{CHADS}_{2}$ Score & 2.1 & 1.3 & 2.1 & 1.3 \\
\hline 0 & 3,444 & $9.0 \%$ & 3,637 & $9.5 \%$ \\
\hline 1 & 10,151 & $26.4 \%$ & 10,266 & $26.7 \%$ \\
\hline 2 & 12,542 & $32.6 \%$ & 12,002 & $31.2 \%$ \\
\hline $3+$ & 12,333 & $32.1 \%$ & 12,565 & $32.7 \%$ \\
\hline $\mathrm{CHA}_{2} \mathrm{DS}_{2}$-VASc Score & 3.2 & 1.7 & 3.2 & 1.8 \\
\hline 0 & 2,326 & $6.1 \%$ & 2,477 & $6.4 \%$ \\
\hline 1 & 3,959 & $10.3 \%$ & 4,008 & $10.4 \%$ \\
\hline 2 & 6,896 & $17.9 \%$ & 6,911 & $18.0 \%$ \\
\hline 3 & 8,748 & $22.7 \%$ & 8,388 & $21.8 \%$ \\
\hline $4+$ & 16,541 & $43.0 \%$ & 16,686 & $43.4 \%$ \\
\hline HAS-BLED Score ${ }^{a}$ & 2.6 & 1.3 & 2.6 & 1.4 \\
\hline 0 & 1,918 & $5.0 \%$ & 1,939 & $5.0 \%$ \\
\hline 1 & 6,101 & $15.9 \%$ & 6,158 & $16.0 \%$ \\
\hline 2 & 11,198 & $29.1 \%$ & 10,950 & $28.5 \%$ \\
\hline $3+$ & 19,253 & $50.0 \%$ & 19,423 & $50.5 \%$ \\
\hline Bleeding history & 6,303 & $16.4 \%$ & 6,393 & $16.6 \%$ \\
\hline Congestive heart failure & 9,210 & $23.9 \%$ & 9,320 & $24.2 \%$ \\
\hline Diabetes mellitus & 12,629 & $32.8 \%$ & 12,501 & $32.5 \%$ \\
\hline
\end{tabular}

\begin{tabular}{|c|c|c|c|c|}
\hline & \multicolumn{2}{|c|}{$\begin{array}{l}\text { Warfarin Cohort } \\
(\mathrm{N}=38,470)\end{array}$} & \multicolumn{2}{|c|}{$\begin{array}{l}\text { Apixaban Cohort } \\
(\mathrm{N}=38,470)\end{array}$} \\
\hline & N/Mean & $\% / S D$ & N/Mean & $\% / S D$ \\
\hline Hypertension & 31,672 & $82.3 \%$ & 31,752 & $82.5 \%$ \\
\hline Renal disease & 7,660 & $19.9 \%$ & 7,628 & $19.8 \%$ \\
\hline Liver disease & 1,653 & $4.3 \%$ & 1,705 & $4.4 \%$ \\
\hline Myocardial infarction & 3,372 & $8.8 \%$ & 3,424 & $8.9 \%$ \\
\hline $\begin{array}{l}\text { Dyspepsia or stomach } \\
\text { discomfort }\end{array}$ & 6,514 & $16.9 \%$ & 6,633 & $17.2 \%$ \\
\hline $\begin{array}{l}\text { Non-stroke/SE peripheral } \\
\text { vascular disease }\end{array}$ & 17,269 & $44.9 \%$ & 17,337 & $45.1 \%$ \\
\hline Stroke/SE & 3,812 & $9.9 \%$ & 3,922 & $10.2 \%$ \\
\hline Transient ischaemic attack & 2,360 & $6.1 \%$ & 2,389 & $6.2 \%$ \\
\hline $\begin{array}{l}\text { Anaemia and coagulation } \\
\text { defects }\end{array}$ & 7,221 & $18.8 \%$ & 7,141 & $18.6 \%$ \\
\hline Alcoholism & 797 & $2.1 \%$ & 809 & $2.1 \%$ \\
\hline \multicolumn{5}{|l|}{ Baseline Medication Use } \\
\hline ACE/ARB & 22,727 & $59.1 \%$ & 22,562 & $58.6 \%$ \\
\hline Amiodarone & 4,174 & $10.9 \%$ & 4,221 & $11.0 \%$ \\
\hline Beta blockers & 23,005 & $59.8 \%$ & 23,111 & $60.1 \%$ \\
\hline H2-receptor antagonist & 1,984 & $5.2 \%$ & 1,992 & $5.2 \%$ \\
\hline Proton pump inhibitor & 10,479 & $27.2 \%$ & 10,636 & $27.6 \%$ \\
\hline Statins & 21,891 & $56.9 \%$ & 21,754 & $56.5 \%$ \\
\hline Anti-platelets & 5,995 & $15.6 \%$ & 6,093 & $15.8 \%$ \\
\hline NSAIDs & 8,953 & $23.3 \%$ & 9,045 & $23.5 \%$ \\
\hline \multicolumn{5}{|l|}{ Apixaban Dose on Index Date } \\
\hline Standard (5 mg) & - & - & 31,926 & $83.0 \%$ \\
\hline Reduced (2.5 mg) & - & - & 6,568 & $17.1 \%$ \\
\hline Follow-up Time (in days) & 199.9 & 193.8 & 179.2 & 163.2 \\
\hline Median & 122.0 & - & 119.0 & - \\
\hline $\begin{array}{l}\text { Follow-up Time (in days) } \\
\text { Restricted to } 1 \text { Year }\end{array}$ & 165.7 & 117.3 & 158.2 & 114.8 \\
\hline Median & 122.0 & - & 119.0 & - \\
\hline
\end{tabular}

SD: standard deviation; SE: systemic embolism; $\mathrm{CHADS}_{2}$ : congestive heart failure, hypertension, age $\geq 75$ years, diabetes mellitus, prior stroke or transient ischaemic attack or thromboembolism; $\mathrm{CHA}_{2} \mathrm{DS}_{2}$-VASC: congestive heart failure, hypertension, age $\geq 75$ years, diabetes mellitus, prior stroke or transient ischaemic attack or thromboembolism, vascular disease, age 65-74 years, sex category; HAS-BLED: hypertension, abnormal renal and liver function, stroke, bleeding, labile INRs (international normalised ratio), elderly, drugs and alcohol; ACE: angiotensin-converting enzyme inhibitor; ARB: angiotensin-receptor blocker; NSAIDs: non-steroidal anti-inflammatory drugs. a as the INR value is not available in the databases, a modified HAS-BLED score was calculated with a range of 0 to 8 . 
receipt date of a prescription for an OAC other than the index therapy, inpatient death, end of continuous medical and pharmacy enrolment, one year post-index date, or the end of study period (September 30, 2015), whichever occurred first. Discontinuation was defined as no evidence of index warfarin or apixaban prescription for 30 days from the last day of supply of the last filled prescription (25). Patients were censored one year post-index date in order to balance the follow-up period between the apixaban and warfarin cohorts.

\section{Statistical methods}

Propensity score matching (PSM) was conducted between the warfarin and apixaban cohorts. Nearest neighbour without replacement with a calliper of 0.01 was used to match the patients (26). Patients were matched 1:1 within each dataset on the propensity scores generated by logistic regressions based on age, gender, geographic region, Charlson Comorbidity Index (CCI) score, baseline bleeding and stroke/SE history, comorbidities, and baseline co-medications. The balance of covariates was checked based on standardised differences with a threshold of $10 \%$ (27). The four datasets were pooled after ensuring the cohorts were balanced.

Cox proportional hazard models with robust sandwich estimates were performed to evaluate the risk of stroke/SE and major bleeding between the two matched cohorts (26). Apixaban or warfarin treatment was included as the independent variable, and no other covariates were included in the model because the cohorts were balanced. The proportional hazards assumption was checked by visual inspection of log-log of the Kaplan-Meier survival curves.

\section{Subgroup analyses}

Subgroup analyses were completed based on initial apixaban dose, age strata, $\mathrm{CHA}_{2} \mathrm{DS}_{2}$-VASc score, HAS-BLED score, and the dataset source. For the dose subgroup analysis, apixaban standard dose $(5 \mathrm{mg})$ and reduced dose $(2.5 \mathrm{mg})$ subgroups were created based on the index apixaban prescription dosage. Each warfarin patient was assigned to one of the two subgroups according to the dose of the apixaban patient she/he matched with. For the age subgroup analysis, patients in the pooled dataset were categorised into three subgroups: $<65,65-74$, and $\geq 75$ years. For the $\mathrm{CHA}_{2} \mathrm{DS}_{2}$-VASc score subgroup analysis, patients were categorised by scores of $<2$, $2-3$, and $\geq 4$; for the HAS-BLED subgroup analysis, patients were categorised by scores of $<3$ and $\geq 3$ (as the INR value is not available in the databases, a modified HAS-BLED score was calculated with a range of 0 to 8). In each subgroup, the balance of baseline characteristics between apixaban and warfarin patients was evaluated. When the standardized difference was $>10 \%$, the covariate was included in the Cox proportional hazards model. In each subgroup analysis, the statistical significance (p-value $<0.10$ ) of the interaction between treatment and the specific subgroup(s) was evaluated.

\section{Sensitivity analysis}

A sensitivity analysis was conducted without restricting the followup period to one year. In this analysis, patients were not censored one year post-index date.

\section{Results \\ Baseline characteristics}

After applying the selection criteria, a total of 115,186 NVAF patients newly initiated on warfarin or apixaban were identified, including 41,867 apixaban and 73,319 warfarin patients. Before PSM, warfarin patients were significantly older and had higher CCI, $\mathrm{CHA}_{2} \mathrm{DS}_{2}$-VASc, and HAS-BLED scores compared to apixaban patients in each of the four datasets. After PSM, a total of 76,940 (38,470 warfarin and 38,470 apixaban) patients were included in the final analysis (14,563 pairs from MarketScan, 7,683 pairs from PharMetrics, 7,894 pairs from Optum, and 8,330 pairs from Humana), and the two matched cohorts were well balanced $(\triangleright$ Figure 1$)$.

The mean age for the matched warfarin and apixaban cohorts was 71 years. The mean $\mathrm{CCI}, \mathrm{CHA}_{2} \mathrm{DS}_{2}$-VASc, and HAS-BLED scores were $2.5,3.2$, and 2.6 , respectively. Approximately $16 \%$ of the patient population had a prior bleed, and about $10 \%$ had stroke/SE during the baseline period. $17 \%$ of patients were prescribed reduced-dose apixaban on the index date. Compared with patients enrolled in the ARISTOTLE trial, patients in this "realworld" study were more likely to be aged $\geq 75$ years and female, had similar mean $\mathrm{CHADS}_{2}$ scores, and had similar percentage of patients with bleeding history (Suppl. Table 2, available online at www.thrombosis-online.com) (9). For the main analysis, we restricted the follow-up to one year, which created a more similar follow-up length between the two cohorts (mean: 166 vs 158 days; median: 122 vs 119 days) ( Table 1$)$.

\section{Stroke/SE}

The incidence of stroke/SE was 2.3 and 3.5 per 100 person-years (PY) for apixaban and warfarin patients, respectively ( Table 2). The cumulative incidence of stroke/SE and major bleeding is shown in $>$ Figure 2. Compared to warfarin, apixaban was associated with a $33 \%$ lower risk of stroke/SE (hazard ratio [HR]: 0.67 , $95 \%$ confidence interval [CI]: 0.59-0.76, $\mathrm{p}<0.001$ ) within one year of treatment initiation and driven by a reduction in the risk of haemorrhagic stroke (HR: $0.70,95 \%$ CI: $0.50-0.99, \mathrm{p}=0.041$ ), ischaemic stroke (HR $0.67,95 \%$ CI $0.58-0.76, \mathrm{p}<0.001$ ), and SE (HR: $0.46,95 \%$ CI: $0.26-0.82, \mathrm{p}=0.008)$ ( $\triangleright$ Figure 3 ).

\section{Major bleeding}

The incidence of major bleeding for apixaban and warfarin patients was 4.5 and 7.5 per $100 \mathrm{PY}$, respectively ( Table 2). Compared to warfarin, apixaban use was associated with a $40 \%$ lower risk of major bleeding (HR: 0.60, 95\% CI: $0.54-0.65$, p $<0.001$ ) 


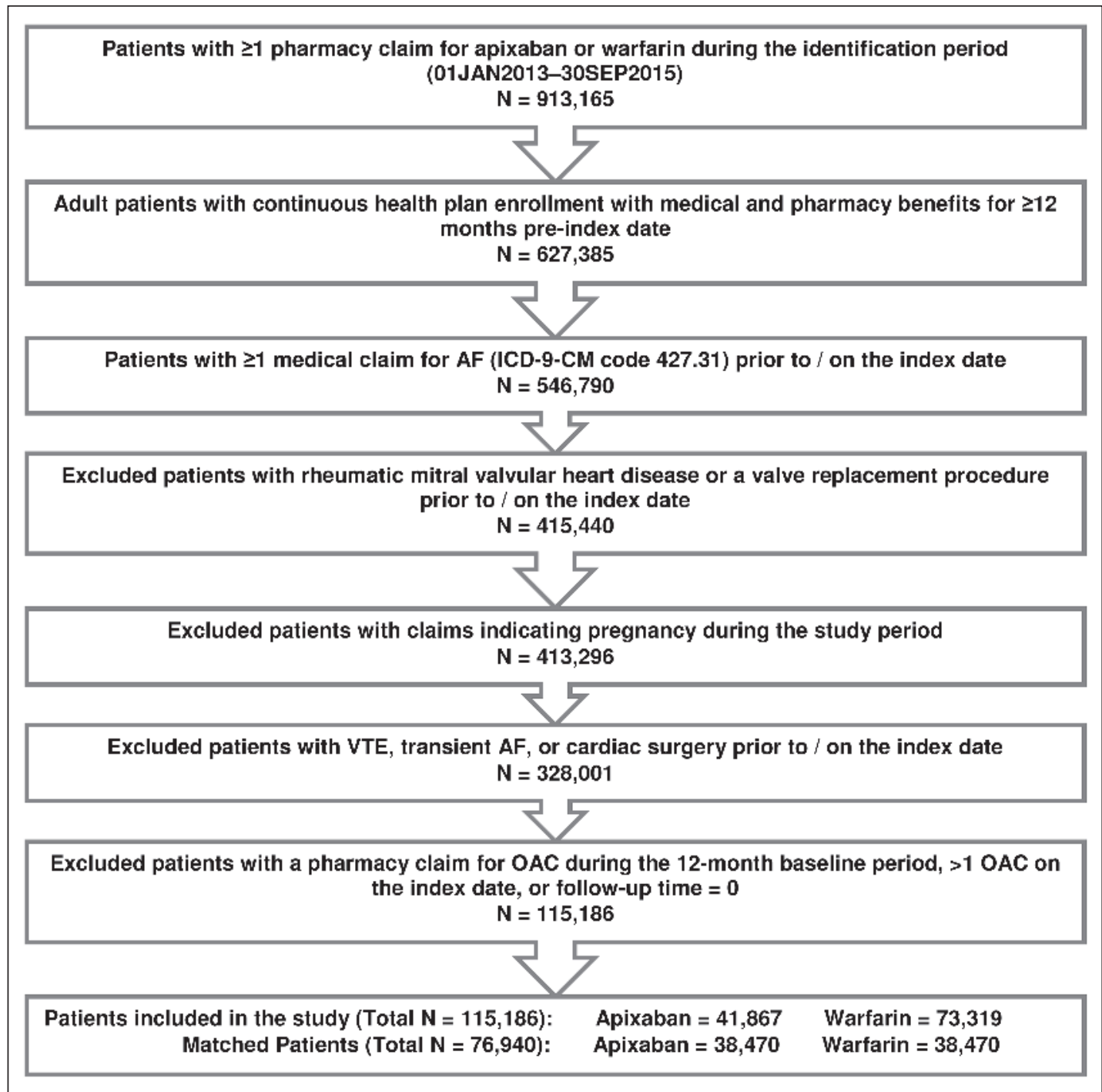

Figure 1: Patient selection criteria. AF: atrial fibrillation; VTE: venous thromboembolism; OAC: oral anticoagulant.

within one year of treatment initiation. This decrease in risk was driven by a reduction in all types of major bleeding, including GI (HR: 0.62, 95\% CI: 0.55-0.71, p<0.001), ICH (HR: 0.64, 95\% CI: $0.50-0.80, \mathrm{p}<0.001$ ), and other major bleeding (HR: 0.57, $95 \% \mathrm{CI}$ : $0.50-0.65, \mathrm{p}<0.001)$ ( $>$ Figure 3 ).

\section{Subgroup analyses}

No significant interaction was found between the treatment and initial apixaban dose in regards to stroke/SE $(\mathrm{p}=0.848)$ and major bleeding $(\mathrm{p}=0.561)$. In the reduced and standard dose subgroups, apixaban was associated with a $34 \%$ (HR: 0.66, 95\% CI: 0.51-0.85) and $32 \%$ (HR: 0.68, 95\% CI: 0.58-0.78) lower risk of stroke/SE, respectively, when compared to warfarin. Reduced and standard dose apixaban patients were associated with a $43 \%$ (HR: 0.57, $95 \%$ CI: $0.47-0.69$ ) and $39 \%$ (HR: 0.61, 95\% CI: 0.55-0.67) lower risk of major bleeding, respectively, when compared to corresponding warfarin patients ( $>$ Figure 4 ).
The results on stroke/SE and major bleeding were consistent across the three age groups. Compared to warfarin, apixaban was associated with a $27 \%$ (HR: $0.73,95 \%$ CI: 0.54-0.98), $20 \%$ (HR: 0.80, 95\% CI: 0.62-1.03), and 38\% (HR: 0.62, 95\% CI: 0.52-0.73) lower risk of stroke/SE among patients aged $<65,65-74$, and $\geq 75$ years, respectively. Among patients aged $<65,65-74$, and $\geq 75$ years, apixaban treatment was associated with a $48 \%$ (HR: 0.52 , 95\% CI: 0.42-0.65), $43 \%$ (HR: 0.57, 95\% CI: 0.48-0.69), and 35\% (HR: 0.65, 95\% CI: 0.57-0.73) lower risk of major bleeding, respectively, when compared to warfarin. No significant interaction on the stroke/SE $(\mathrm{p}=0.226)$ and major bleeding $(\mathrm{p}=0.181)$ outcomes was found between treatment and age ( $>$ Figure 4 ).

Among patients with $\mathrm{CHA}_{2} \mathrm{DS}_{2}$-VASc scores $<2,2-3$, and $\geq 4$, apixaban treatment was associated with a $16 \%$ (HR: 0.84, $95 \% \mathrm{CI}$ : $0.45-1.58$ ), $39 \%$ (HR: 0.61, 95\% CI: $0.47-0.80$ ), and $31 \%$ (HR: 0.69, 95\% CI: $0.59-0.80)$ lower risk of stroke/SE, respectively, when compared to warfarin. Compared to warfarin, apixaban was associated with a $48 \%$ (HR: 0.52, 95\% CI: 0.35-0.77), 49\% (HR: 
Table 2: Number of patients with event and incidence rates during one-year follow-up period.

\begin{tabular}{|c|c|c|c|c|}
\hline & \multicolumn{2}{|l|}{$\begin{array}{l}\text { Warfarin Cohort } \\
(\mathrm{N}=38,470)\end{array}$} & \multicolumn{2}{|l|}{$\begin{array}{l}\text { Apixaban Cohort } \\
(\mathrm{N}=38,470)\end{array}$} \\
\hline & Patients with Event & Incidence Rate & Patients with Event & Incidence Rate \\
\hline Stroke/SE & 609 & 3.47 & 394 & 2.34 \\
\hline Ischaemic Stroke & 515 & 2.93 & 332 & 1.97 \\
\hline Haemorrhagic Stroke & 82 & 0.46 & 55 & 0.33 \\
\hline SE & 38 & 0.21 & 17 & 0.10 \\
\hline Major Bleeding & 1,303 & 7.47 & 753 & 4.49 \\
\hline $\mathrm{ICH}$ & 183 & 1.03 & 111 & 0.66 \\
\hline GI Bleeding & 630 & 3.58 & 379 & 2.25 \\
\hline Other Bleeding & 582 & 3.31 & 320 & 1.90 \\
\hline
\end{tabular}

Event rates are shown per 100 person-years. SE: systemic embolism; ICH: intracranial haemorrhage; GI: gastrointestinal.
0.51, $95 \%$ CI: 0.44-0.61), and 34\% (HR: 0.66, 95\% CI: 0.59-0.73) lower risk of major bleeding among patients with $\mathrm{CHA}_{2} \mathrm{DS}_{2}$-VASc scores $<2,2-3$, and $\geq 4$, respectively. There was no significant interaction for stroke/SE between treatment and $\mathrm{CHA}_{2} \mathrm{DS}_{2}$-VASc scores $(p=0.587)$, but a significant interaction for major bleeding was found $(\mathrm{p}=0.041)$.

No significant interaction was found between treatment and HAS-BLED score in regards to stroke/SE $(\mathrm{p}=0.439)$ and major bleeding $(\mathrm{p}=0.995)$. Compared to warfarin, apixaban was associated with a $27 \%$ (HR: $0.73,95 \%$ CI: $0.56-0.94)$ and $35 \%$ (HR: $0.65,95 \%$ CI: $0.56-0.75)$ lower risk of stroke/SE in the low and high major bleeding risk subgroups (HAS-BLED scores $<3$ and $\geq 3$ ). Apixaban patients with HAS-BLED scores $<3$ (HR: 0.59, $95 \%$ CI: $0.50-0.70$ ) and $\geq 3$ (HR: 0.59 , 95\% CI: $0.53-0.66$ ) had a significantly lower risk of major bleeding compared to warfarin.

The interaction between treatment and different datasets was tested, and no significant interaction was found (stroke/SE: $p=0.796$; major bleeding: $p=0.199$ ). The stroke/SE results within each dataset were consistent with the overall results, with apixaban associated with significantly lower risk of stroke/SE versus warfarin: $\mathrm{HR}=0.65$ (95\% CI: 0.52-0.82) in MarketScan, HR=0.70 (95\% CI: 0.50-0.98) in PharMetrics, HR=0.62 (95\% CI: 0.48-0.81) in Optum, and $\mathrm{HR}=0.73$ (95\% CI: 0.59-0.91) in Humana. Major bleeding results within each dataset were also consistent with the overall results, with apixaban associated with significantly lower risk of major bleeding versus warfarin: $\mathrm{HR}=0.63$ (95\% CI: 0.54-0.74) in MarketScan, HR=0.47 (95\% CI: 0.37-0.60) in PharMetrics, HR=0.61 (95\% CI: 0.49-0.74) in Optum, and HR=0.63 (95\% CI: 0.54-0.74) in Humana ( Figure 4).

\section{Sensitivity analysis}

In order to utilise a longer follow-up period, we conducted a sensitivity analysis using the entire follow-up period. The mean followup period for warfarin patients was longer than that for apixaban patients (200 vs 179 days). Although most patients had a follow-up less than one year, the maximum follow-up for warfarin patients was 2.8 years and 2.6 years for apixaban patients. Over the entire follow-up, patients treated with apixaban had a significantly lower risk of stroke/SE (HR: 0.67; 95\% CI: 0.60-0.76, p<0.001) and major bleeding (HR: $0.61 ; 95 \% \mathrm{CI}$ : $0.56-0.66, \mathrm{p}<0.001)$ compared to warfarin.

\section{Discussion}

This retrospective "real-world" study examined and compared the risk of stroke/SE and major bleeding among NVAF patients who newly initiated warfarin and apixaban treatment using pooled data from four large US national claims datasets. We show that after PSM, apixaban initiation was associated with significant risk reductions in stroke/SE and major bleeding compared to warfarin initiation. These benefits were consistent across apixaban dose regimens, various high-risk patient subgroups, and database sources.

This is by far the largest "real-world" evaluation on effectiveness and safety of apixaban, with a sample size $(76,940)$ more than four times that of the apixaban registrational trial ARISTOTLE (9,120 apixaban patients and 9,081 warfarin patients) (9). The effectiveness and safety results observed in this "real-world" study were generally consistent with those of the ARISTOTLE trial, where apixaban was superior to warfarin in reducing the risk of stroke/SE (HR: 0.79; 95\% CI: 0.66-0.95, p<0.001) with fewer major bleeding events (HR: 0.69; $95 \%$ CI: $0.60-0.80, \mathrm{p}<0.001)(9)$. In the current study, the different types of stroke/SE - including ischaemic stroke, haemorrhagic stroke, and SE - were all significantly lower for apixaban compared to warfarin treatment; in the ARISTOTLE trial, only haemorrhagic stroke events were significantly lower for patients treated with apixaban, and ischaemic stroke and SE were numerically lower for apixaban patients. Similarly, the different types of major bleeding in the current study including ICH, GI bleeding, and other major bleeding - were significantly lower for patients treated with apixaban than those treated with warfarin. The ARISTOTLE trial also reported a significantly lower risk of ICH and major bleeding at other sites for apixaban compared to warfarin. However, patients on apixaban 


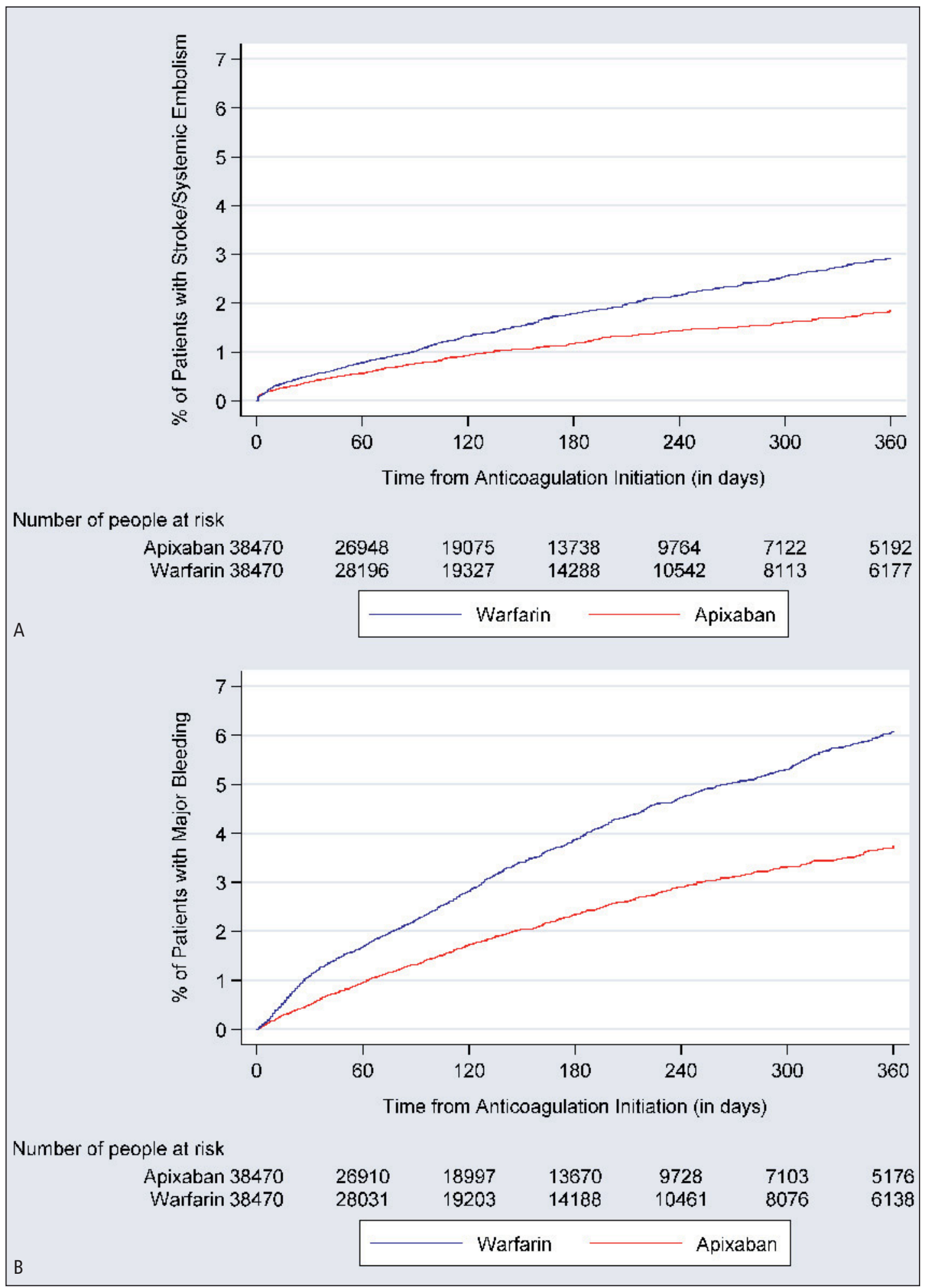

Figure 2: Cumulative incidence of stroke/ systemic embolism (A) and major bleeding (B).

treatment in ARISTOTLE had numerically lower - but not significantly different - rates of GI bleeding compared to those treated with warfarin (9).

In our study, subgroup analyses revealed no significant interaction between treatment and apixaban dosage, age, HAS-BLED score, or datasets in regards to the stroke/SE and major bleeding outcomes. Consistent with the main analysis, each subgroup analysis showed results of lowered stroke/SE and major bleeding risk associated with apixaban versus warfarin treatment. The results of the dosage, age, and bleeding risk stratification score sub- 


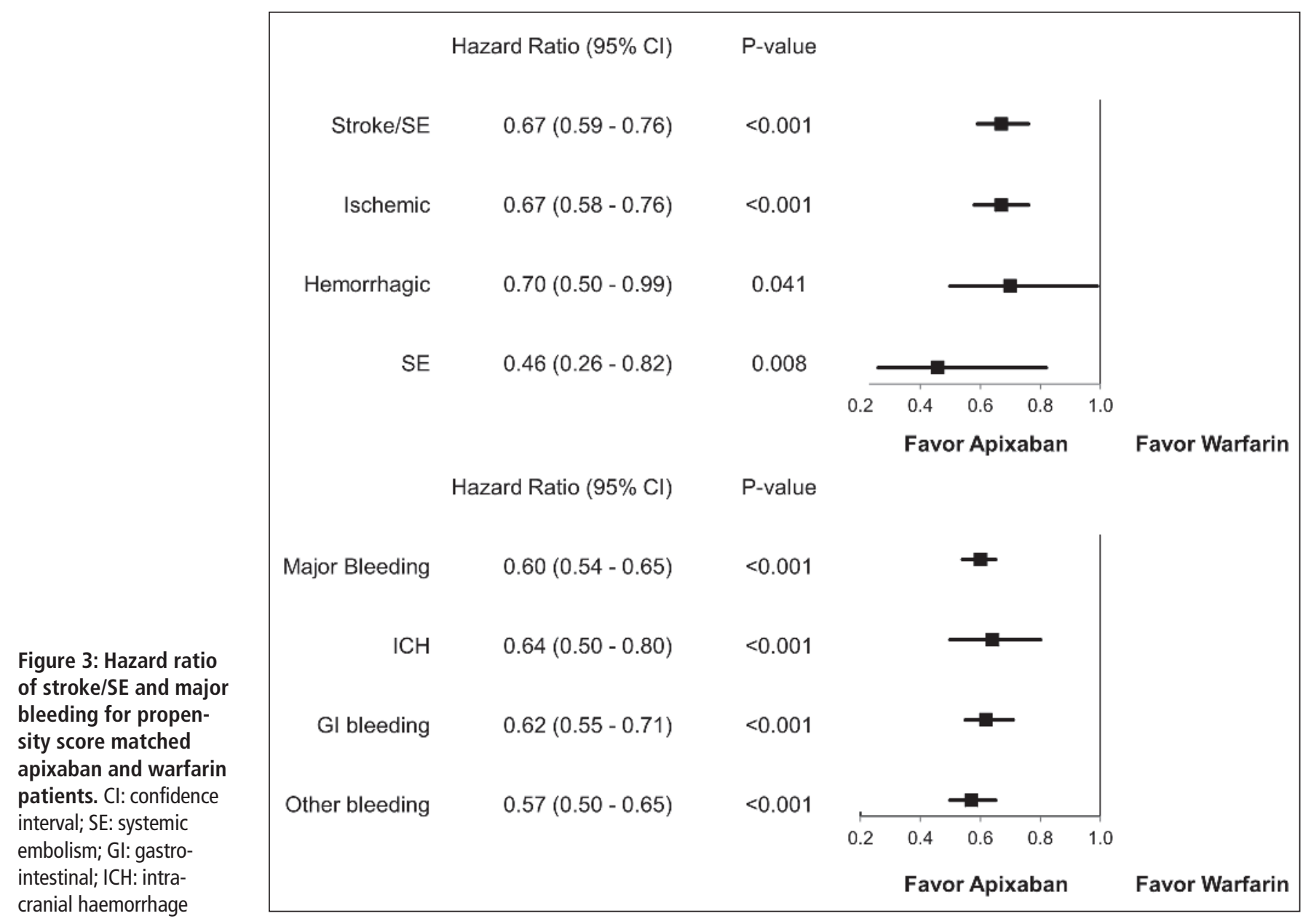

group analyses were also similar to those in the ARISTOTLE trial, which did not find significant interaction effects across those subgroups either $(9,28,29)$.

For the $\mathrm{CHA}_{2} \mathrm{DS}_{2}$-VASc subgroup analysis, no significant interaction was detected in the analysis of stroke/SE, but the interaction term was significant in the analysis of major bleeding. Across all $\mathrm{CHA}_{2} \mathrm{DS}_{2}$-VASc scores, apixaban patients had a significantly lower risk of stroke/SE and major bleeding compared to warfarin. In ARISTOTLE trial subgroup analysis on $\mathrm{CHA}_{2} \mathrm{DS}_{2}$-VASc scores, a significant interaction effect was not found for either stroke/SE or major bleeding (29).

The follow-up period was limited to one year in the current study, given that most patients $(85 \%)$ had a follow-up shorter than one year. Additionally, apixaban entered the market recently, and patients treated with apixaban tended to have a shorter follow-up than those treated with warfarin. Restricting follow-up to one year allowed the duration to be more balanced between the apixaban and warfarin cohorts. After restricting the follow-up to one year, the average follow-up time was approximately 5-5.5 months in both cohorts. A sensitivity analysis was completed using the entire follow-up period, and the results were consistent. Patients treated with apixaban had a significantly lower risk of stroke/SE (HR: 0.67; 95\% CI: 0.60-0.76, p<0.001) and major bleeding (HR: 0.61;
95\% CI: $0.56-0.66, \mathrm{p}<0.001)$ compared to those treated with warfarin.

The results from the current study are also consistent with other "real-world" observational studies evaluating ARISTOTLE primary endpoints (stroke/SE and major bleeding) with data from US clinical practice $(30,31)$. In a recent publication using administrative claims data from Optum Labs Data Warehouse, 7,695 apixaban and 7,695 warfarin patients were matched. The HR for apixaban use compared to warfarin use was 0.67 (95\% CI: 0.46-0.98) for stroke/SE and 0.45 (95\% CI: 0.34-0.59) for major bleeding (30). This publication also found that haemorrhagic stroke, ICH, and GI bleeding were significantly lower among apixaban patients compared to warfarin patients. However, this paper did not find a significant difference for ischaemic stroke (HR: 0.83, 95\% CI: $0.53-1.29$ ), which may have been due to the smaller sample size (30). Consistent with the current study, another study evaluating major bleeding risk using MarketScan Commercial and Medicare Supplemental data found that apixaban patients had a $47 \%$ lower risk of major bleeding among all users and a $45 \%$ lower risk of major bleeding among standard dose users when compared to warfarin patients (31).

This study pooled four large claims datasets and evaluated the effectiveness and safety outcomes comparing apixaban and warfa- 


\begin{tabular}{|c|c|c|c|}
\hline & Hazard Ratio $(95 \% \mathrm{Cl})$ & P-value ${ }^{a}$ & \\
\hline Dose $^{b}$ & & 0.848 & \\
\hline Reduced & $0.66(0.51-0.85)$ & & \\
\hline Standard & $0.68(0.58-0.78)$ & & \\
\hline Age $^{c}$ & & 0.226 & \\
\hline$<65$ & $0.73(0.54-0.98)$ & & \\
\hline $65-74$ & $0.80(0.62-1.03)$ & & \\
\hline$\geq 75$ & $0.62(0.52-0.73)$ & & \\
\hline $\mathrm{CHA}_{2} \mathrm{DS}_{2}-\mathrm{VASc}$ & & 0.587 & \\
\hline $0-1$ & $0.84(0.45-1.58)$ & & \\
\hline $2-3$ & $0.61(0.47-0.80)$ & - & \\
\hline$\geq 4$ & $0.69(0.59-0.80)$ & $\rightarrow-$ & \\
\hline HAS-BLED & & 0.439 & \\
\hline$<3$ & $0.73(0.56-0.94)$ & & \\
\hline$\geq 3$ & $0.65(0.56-0.75)$ & $\rightarrow$ & \\
\hline Dataset & & 0.796 & \\
\hline MarketScan & $0.65(0.52-0.82)$ & $\rightarrow$ & \\
\hline PharMetrics & $0.70(0.50-0.98)$ & $\longrightarrow$ & \\
\hline Optum & $0.62(0.48-0.81)$ & $\longrightarrow$ & \\
\hline Humana & $0.73(0.59-0.91)$ & $\rightarrow$ & \\
\hline & & $\begin{array}{llll}0.2 & 0.4 & 0.6 & 0.8\end{array}$ & $\begin{array}{llll}1 & 1.2 & 1.4 & 1.6\end{array}$ \\
\hline A & & Favor Apixaban & Favor Warfarin \\
\hline
\end{tabular}

Figure 4: Hazard ratio of stroke/SE (A) and major bleeding (B) according to subgroups in propensity score matched apixaban and warfarin patients. Cl: confidence interval. ap-value in the figure is for interaction. bage, gender, geographical region, $\mathrm{CCl}$, $\mathrm{CHA}_{2} \mathrm{DS}_{2}$-VASC, HAS$B L E D$, congestive heart failure, hypertension, renal disease, baseline proton pump inhibitor use, and baseline antiplatelets use were included in the model. ${ }^{\mathrm{C}} \mathrm{Cl}$ was included in the model. rin. By pooling the datasets, this study increased the statistical power to evaluate the differences in the entire population and among several subgroup populations. Furthermore, since four datasets were pooled, the generalisability of these results to the US population is substantially greater than prior, single-source studies. Findings from this study may inform the discussion of risks of stroke and major bleeding in the shared decision making process between healthcare providers and individual AF patients in selecting appropriate antithrombotic therapy (5).

\section{Limitations}

Our study has several limitations. First, only associations could be concluded from this retrospective observational study. Although cohorts were matched through PSM, potential residual confounders - such as over-the-counter use of aspirin and dose change for the warfarin treatment - exist. Due to the nature of the data, no laboratory results - such as creatinine clearance or INR were available; time in therapeutic range information, which measures the quality of anticoagulation control among warfarin patients, was also unavailable. Second, given the nature of claims data, diagnoses were identified through ICD-9-CM codes and drug prescriptions were identified through prescription claims. Missing values, coding errors, and lack of clinical accuracy may have introduced bias into the study. Third, deaths that occurred outside the hospital setting are not captured; therefore, we were not able to accurately assess mortality. Death was obtained from hospital discharge records and reasons for mortality are not available, which may have biased the time-to-event analysis. Fourth, although some of the datasets contain information from different insurance plans that do not overlap at the plan level, others are employer-based claims datasets which may contain duplicate patient records when pooled together; however, the number of such duplicates is likely to be small - based on a published estimate of $0.5 \%$ - and therefore unlikely to have any important effect on results (21). Fifth, only apixaban and warfarin were included in this analysis, and comparisons between the use of warfarin and other NOACs will be conducted in future analyses. The apixaban and warfarin comparison was conducted first because few studies have examined the safety and effectiveness of apixaban due to its recent 


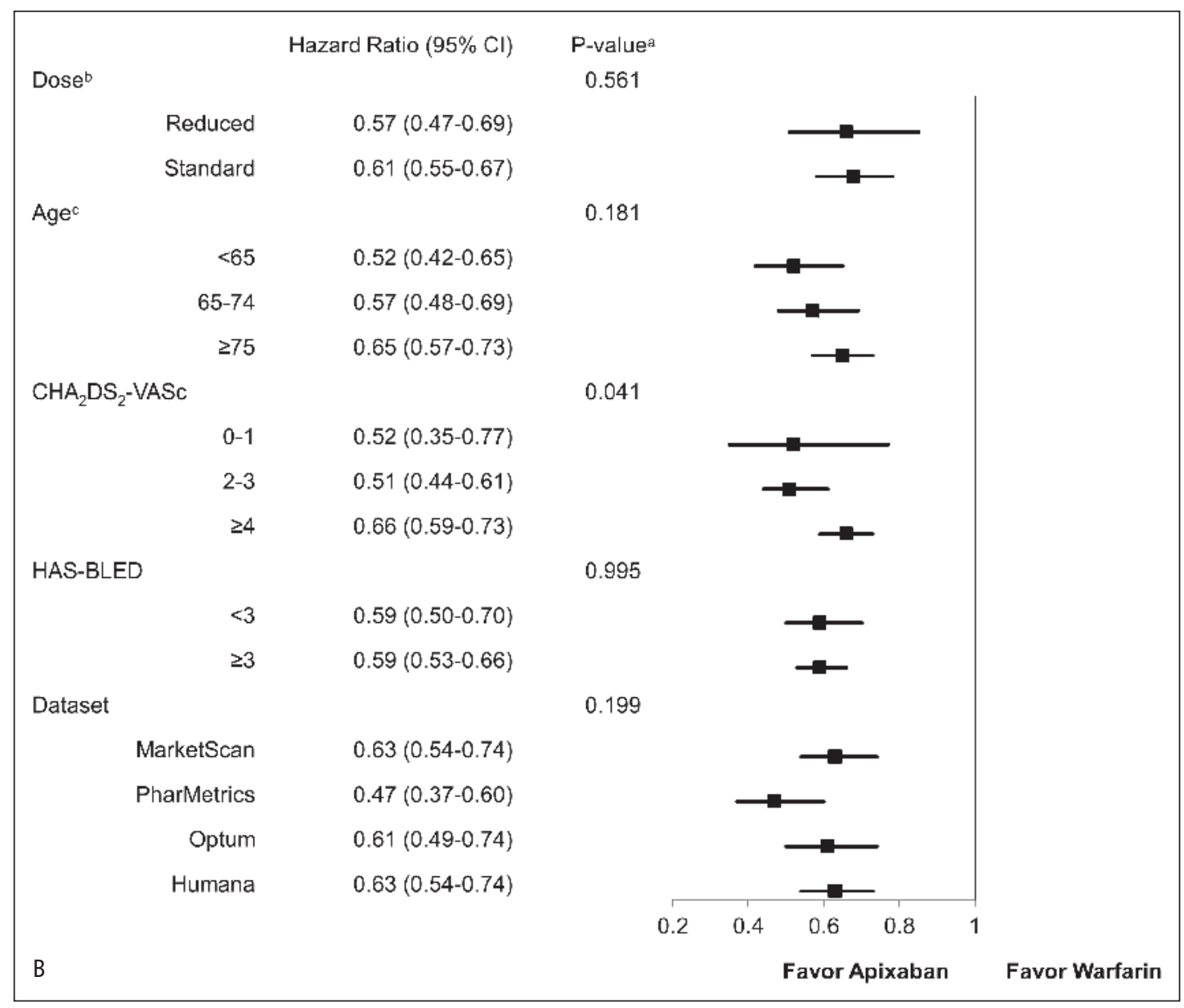

market entry. Prior studies evaluating the effectiveness of apixaban may have had limited statistical power (30); however, one of the main strengths of this study is the large sample size and sufficient statistical power necessary to evaluate both effectiveness and safety. Sixth, this study only included treatment-naïve apixaban and warfarin patients because a comparison between patients who switched from warfarin to apixaban and patients who continued on warfarin could be potentially confounded (the reason for a switch could be poor quality of INR control, which cannot be measured in the data source). Last, the mean follow-up time in our study was only 5-5.5 months. As more data becomes available, we plan to conduct further analyses using a longer follow-up period.

Furthermore, several factors may contribute to some of the differences between this "real-world" study and clinical trials (32). First, the stroke/SE and major bleeding events in this "real-world" study were based on claims data; in the ARISTOTLE trial, these events were adjudicated. In contrast to the clinical trial, this "realworld" study applied less-strict selection criteria and included a larger patient sample size. Additionally, warfarin management in the "real-world" may not be as good as that in the clinical trial, which may lead to higher rates of ischaemic stroke and GI bleeding for patients treated with warfarin.
In conclusion, this "real-world" retrospective study offers the largest sample of comparisons between apixaban and warfarin patients to date. After PSM adjustment for differences in patient characteristics, NVAF patients treated with apixaban had a significantly lower risk of stroke/SE and major bleeding compared to patients treated with warfarin. Importantly, these benefits were con-

\section{What is known about this topic?}

- Apixaban is the only non-VKA oral anticoagulant to show risk reduction in both stroke/systemic embolism (SE) and major bleeding compared to warfarin in its Phase 3 trial.

- Due to apixaban's recent market entry, prior "real-world" studies were limited by sample size and follow-up duration.

\section{What does this paper add?}

- Apixaban initiation is associated with a significantly lower risk of stroke/SE and major bleeding compared to warfarin initiation.

- Subgroup analyses (apixaban dosage, age strata, $\mathrm{CHA}_{2} \mathrm{DS}_{2}$-VASC or HAS-BLED score strata, or dataset source) all show consistently lower risks of stroke/SE and major bleeding associated with apixaban as compared to warfarin treatment. 
sistent across apixaban dose regimens, various high-risk patient subgroups, and database sources, providing complementary "realworld" data to the clinical trial results.

\section{Conflicts of interest}

Drs. Li, Hamilton, Gupta, Friend, Nadkarni, and Pan are employees of Bristol-Myers Squibb Company with ownership of stocks in Bristol-Myers Squibb Company. Dr. Deitelzweig is a consultant for Bayer/Janssen, Bristol-Myers Squibb Company/Pfizer Inc., Daiichi-Sankyo, Portola, and Boehringer Ingelheim, and has been on the speakers' bureau for Janssen, Bristol-Myers Squibb Company/Pfizer Inc., and Boehringer Ingelheim. Dr. Keshishian is an employee of STATinMED Research, a paid consultant to Bristol-Myers Squibb Company and Pfizer, Inc. in connection with conducting this study. Drs. Luo and Mardekian are employees of Pfizer Inc., with ownership of stocks in Pfizer Inc. Dr. Horblyuk is a former employee of Pfizer Inc., with ownership of stocks in Pfizer Inc. Professor Lip has served as a consultant for Bayer/ Janssen, Merck, Sanofi, Bristol-Myers Squibb Company/Pfizer Inc., Daiichi-Sankyo, Biotronik, Medtronic, Portola, and Boehringer Ingelheim, and has been on the speakers' bureau for Bayer, Bristol-Myers Squibb Company/Pfizer Inc., Boehringer Ingelheim, Daiichi-Sankyo, and Medtronic. Chris Haddlesey, Qisu Zhang, and Elizabeth Vivier of STATinMED provided medical writing and editorial support which was funded by Bristol-Myers Squibb and Pfizer. Statistical programming for this study was provided by Juan Du of STATinMED Research and funded by Pfizer Inc. and Bristol-Myers Squibb Company.

\section{References}

1. Wolf PA, Abbott RD, Kannel WB. Atrial fibrillation as an independent risk factor for stroke: the Framingham Study. Stroke 1991; 22: 983-988.

2. Colilla S, Crow A, Petkun W, et al. Estimates of current and future incidence and prevalence of atrial fibrillation in the U.S. adult population. Am J Cardiol 2013; 112: 1142-1147.

3. Hart RG, Pearce LA, Aguilar MI. Meta-analysis: antithrombotic therapy to prevent stroke in patients who have nonvalvular atrial fibrillation. Ann Intern Med 2007; 146: 857-867.

4. Sam C, Massaro JM, D'Agostino RB Sr, et al. Warfarin and aspirin use and the predictors of major bleeding complications in atrial fibrillation (the Framingham Heart Study). Am J Cardiol 2004; 94: 947-951.

5. January CT, Wann LS, Alpert JS, et al. 2014 AHA/ACC/HRS guideline for the management of patients with atrial fibrillation: a report of the American College of Cardiology/American Heart Association Task Force on practice guidelines and the Heart Rhythm Society. Circulation 2014; 130: e199-e267.

6. Nutescu E, Chuatrisorn I, Hellenbart E. Drug and dietary interactions of warfarin and novel oral anticoagulants: an update. J Thromb Thrombolysis 2011; 31: 326-343.

7. Connolly SJ, Ezekowitz MD, Yusuf S, et al. Dabigatran versus warfarin in patients with atrial fibrillation. N Engl J Med 2009; 361: 1139-1151.

8. Patel MR, Mahaffey KW, Garg J, et al. Rivaroxaban versus warfarin in nonvalvular atrial fibrillation. N Engl J Med 2011; 365: 883-891.

9. Granger CB, Alexander JH, McMurray JJ, et al. Apixaban versus warfarin in patients with atrial fibrillation. New Engl J Med. 2011; 365: 981-992.

10. Giugliano RP, Ruff CT, Braunwald E, et al. Edoxaban versus warfarin in patients with atrial fibrillation. N Engl J Med 2013; 369: 2093-2104.

11. Bristol-Meyers Squibb Company and Pfizer Inc. Eliquis (apixaban) U.S. Full Prescribing Information. Available at http://packageinserts.bms.com/pi/pi_eliquis.pdf. Accessed January 29, 2017.
12. Hansen LG, Chang S. Health research data for the real world: the Marketscan databases. Available at truvenhealth.com/portals/0/assets/

PH_11238_0612_TEMP_MarketScan_WP_FINAL.pdf. Published July 2011; Accessed January 26, 2017.

13. IMS Health. PharMetrics Plus Data Dictionary. Available at tri.uams.edu/ files/2015/08/Pharmetrics-Plus-Data-Dictionary-Jan-2013.pdf. Accessed January 26, 2017.

14. Optum. Clinformatics Data Mart. Available at https://www.optum.com/con tent/dam/optum/resources/productSheets/Clinformatics_for_Data_Mart.pdf Published 2014; Accessed January 26, 2016.

15. Michels SL, Uribe C. The Humana database - a data source for pharmacoepidemiologic research. [Presentation] 28th Annual International Conference for Pharmacoepidemiology and Therapeutic Risk Management, Barcelona, Spain. 23rd-26th August 2012.

16. Pelton SI, Weycker D, Farkouh RA, et al. Risk of pneumococcal disease in children with chronic medical conditions in the era of pneumococcal conjugate vaccine. Clin Infect Dis 2014; 59: 615-623.

17. Weycker D, Li X, Barron R, et al. Importance of risk factors for febrile neutropenia among patients receiving chemotherapy regimens not classified as high-risk in guidelines for myeloid growth factor use. J Natl Compr Canc Netw 2015; 13: 979-986.

18. Sun P, Kohrman M, Liu J, et al. Outcomes of resecting subependymal giant cell astrocytoma (SEGA) among patients with SEGA-related tuberous sclerosis complex: a national claims database analysis. Curr Med Res Opin 2012; 28 : 657-663.

19. Guérin A, Sasane M, Zhang J, et al. Brain metastases in patients with ALK+ non-small cell lung cancer: clinical symptoms, treatment patterns and economic burden. J Med Econ 2015; 18: 312-322.

20. Seeger JD, Bykov K, Bartels DB, et al. Safety and effectiveness of dabigatran and warfarin in routine care of patients with atrial fibrillation. Thromb Haemost 2015; 114: 1277-1289.

21. Broder MS, Neary MP, Chang E, et al. Treatments, complications, and healthcare utilization associated with acromegaly: a study in two large United States databases. Pituitary 2014; 17: 333-341.

22. Jensen PN, Johnson K, Floyd J, et al. Identifying atrial fibrillation from electronic medical data: a systematic review. Pharmacoepidemiol Drug Saf 2012; 21: 141-147.

23. Thigpen JL, Dillon C, Forster KB, et al. Validity of international classification of disease codes to identify ischaemic stroke and intracranial hemorrhage among individuals with associated diagnosis of atrial fibrillation. Circ Cardiovasc Qual Outcomes 2015; 8: 8-14.

24. Cunningham A, Stein CM, Chung CP, et al. An automated database case definition for serious bleeding related to oral anticoagulant use. Pharmacoepidemiol Drug Saf 2011; 20: 560-566.

25. Pan X, Kachroo S, Liu X, et al. Real world discontinuation among early users of apixaban, dabigatran, rivaroxaban or warfarin among atrial fibrillation patients newly initiated on anticoagulation therapy: tell of first 200 days. Eur Heart J 2014; 35: 897.

26. Austin PC. The use of propensity score methods with survival or time-to-event outcomes: reporting measures of effect similar to those used in randomized experiments. Stat Med 2014; 33: 1242-1258.

27. Austin PC. Balance diagnostics for comparing the distribution of baseline covariates between treatment groups in propensity-score matched samples. Stat Med 2009; 28: 3083-3107.

28. Halvorsen S, Atar D, Yang H, et al. Efficacy and safety of apixaban compared with warfarin according to age for stroke prevention in atrial fibrillation: observations from the ARISTOTLE trial. Eur Heart J 2014; 35: 1864-1872.

29. Lopes RD, Al-Khatib SM, Wallentin L, et al. Efficacy and safety of apixaban compared with warfarin according to patient risk of stroke and bleeding in atrial fibrillation: a secondary analysis of a randomised controlled trial. Lancet 2012; 380: 1749-1758.

30. Yao X, Abraham NS, Sangaralingham LR, et al. Effectiveness and safety of dabigatran, rivaroxaban, and apixaban versus warfarin in nonvalvular atrial fibrillation. J Am Heart Assoc 2016; 5: e003725.

31. Lip GY, Keshishian A, Kamble S, et al. Real-world comparison of major bleeding risk among non-valvular atrial fibrillation patients initiated on apixaban, dabigatran, rivaroxaban, or warfarin. Thromb Haemost 2016; 116: 975-986.

32. Freedman B, Lip GY. "Unreal world" or "real world" data in oral anticoagulant treatment of atrial fibrillation. Thromb Haemost 2016; 116: 587-589. 